\title{
Heterogeneity in structurally arrested hard spheres
}

\author{
ZI-QI Li ${ }^{1(a)}$, ZHAO-YAN Sun ${ }^{1(a)}$, LI-JiA AN ${ }^{1(b)}$ and ZHEN-GANG WANG ${ }^{2(c)}$ \\ ${ }^{1}$ State Key Laboratory of Polymer Physics and Chemistry, Changchun Institute of Applied Chemistry, \\ Chinese Academy of Sciences, Graduate School of the Chinese Academy of Sciences - Changchun 130022, PRC \\ ${ }^{2}$ Division of Chemistry and Chemical Engineering, California Institute of Technology - Pasadena, CA 91125, USA
}

received 17 July 2008; accepted 9 September 2008

published online 8 October 2008

PACS 61.43.-j- Disordered solids

PACS $61.20 . \mathrm{Ne}$ - Structure of simple liquids

PACS 64.70.Q- - Theory and modeling of the glass transition

\begin{abstract}
When cooled or compressed sufficiently rapidly, a liquid vitrifies into a glassy amorphous state. Vitrification in a dense liquid is associated with jamming of the particles. For hard spheres, the density and degree of order in the final structure depend on the compression rate: simple intuition suggests, and previous computer simulation demonstrates, that slower compression results in states that are both denser and more ordered. In this work, we use the Lubachevsky-Stillinger algorithm to generate a sequence of structurally arrested hard-sphere states by varying the compression rate. We find that while the degree of order, as measured by both bondorientation and translation order parameters, increases monotonically with decreasing compression rate, the density of the arrested state first increases, then decreases, then increases again, as the compression rate decreases, showing a minimum at an intermediate compression rate. Examination of the distribution of the local order parameters and the distribution of the root-mean-square fluctuation of the particle positions, as well as direct visual inspection of the arrested structures, reveal that they are structurally heterogeneous, consisting of disordered, amorphous regions and locally ordered crystal-like domains. In particular, the low-density arrested states correspond with many interconnected small crystal clusters that form a polycrystalline network interspersed in an amorphous background, suggesting that jamming by the domains may be an important mechanism for these states.
\end{abstract}

Copyright (c) EPLA, 2008

Solidification of a liquid - either into amorphous glass [1] or periodic crystal [2] - is a ubiquitous phenomenon in nature and in numerous applications, whose mechanism remains one of the most challenging questions in the physical sciences. Intuitively, slow cooling or compression leads to a perfect crystal, while an amorphous or glassy state is obtained upon rapid cooling or compression, the structural mechanism for the latter being jamming of the particles which prevents their relaxation to the equilibrium crystalline state [3]. As the simplest system that exhibits both jamming and crystallization, the hard-sphere model provides an excellent platform for studying structures of liquids, glasses, granular materials, and random media.

An important recent insight in the study of hard-sphere packing is the clarification of the so-called random close packing (RCP). Torquato et al. [4,5] showed that the

\footnotetext{
(a) ZQL and ZYS contributed equally to this work.

(b) E-mail: 1jan@ciac.jl.cn

(c) E-mail: zgw@caltech.edu
}

notion of RCP as the maximum attainable density with a unique value in a random collection of spheres was ill-defined. These authors proposed instead the concept of maximally random jammed state (MRJ), corresponding to a state that minimizes an appropriately defined order parameter among all statistically homogeneous and isotropic jammed structures. Using an algorithm due to Lubachevsky and Stillinger [6] for increasing the sphere diameters linearly in time (mimicking the compression of the spheres), these authors found that the density of the final jammed state increases monotonically with decreasing rate, with an attendant increase in the structural order, as measured by a bond-orientation order parameter and a translation order parameter.

In this letter, we show that the density of a structurally arrested state obtained by compression of hard spheres can in fact be a non-monotonic function of the compression rate, that increased order does not necessarily result in higher packing density for an arrested state, and that the structure of the arrested states is highly heterogeneous. 
We draw these conclusions from results obtained using molecular dynamics simulation with the LubachevskyStillinger algorithm [6], similar to the method used in refs. $[4,5]$. In this algorithm, regular molecular dynamics for hard-sphere collisions is combined with the growth of the sphere diameter in a constant volume with a dimensionless linear growth rate

$$
\Gamma=\frac{\mathrm{d} \sigma}{\mathrm{d} t} \sqrt{\frac{m}{k T}}
$$

where $m$ is the mass of a sphere, $k$ Boltzmann's constant, and $T$ the absolute temperature. The system is considered structurally arrested when the spheres can no longer grow as determined by the relative change in the sphere diameter from previous collision steps, $\Delta d$, defined as

$$
\Delta d=\frac{1}{N_{c}} \sum_{i=2}^{N_{c}} \frac{\sigma_{i}-\sigma_{i-1}}{\sigma_{i}},
$$

where $\sigma_{i}$ is the diameter of hard sphere in the $i$-th step, and $N_{c}$ is the number of collision steps used to calculate an average $\Delta d$. In our simulation we use $N_{c}=10^{4}$ and assume growth is halted when $\Delta d<5 \times 10^{-9}$.

We note that for hard spheres or disks there exists a hierarchy of jammed configurations, which have been classified as locally, collectively, and strictly jammed [7]. A rigorous and practical algorithm based on linear programming has been developed to test whether a hard disk or sphere packing is jammed according to the three categories [8]. Our focus here, however, is not the creation of particular jammed states according to the stringent geometric jamming criteria. Rather our interest is the examination of the local packing and degrees of order when hard spheres become structurally arrested (on macroscopic scale) under nonequilibrium conditions. Our method is just a simple, intuitive, physically sensible and consistent, means for creating structurally arrested state that mimic those obtained in fast compression or cooling of a liquid. To avoid possible confusion, we refer to the states created by our method as "structurally arrested states" or simply "arrested states" rather than "jammed states". Our arrested states allow caged "rattlers".

We have performed simulations for several values of compression rate in the range $[0.05,0.0008]$ on a system containing 2048 spheres. The number of spheres in our work is four times the number used in refs. [4,5]; this difference is significant since some of the structural features reported here can only be captured when the system is sufficiently large. All simulations begin with an equilibrated liquid at volume fraction $\phi=0.3$.

Figure 1 shows the final packing fraction of the structurally arrested states as a function of inverse of the compression rate $\Gamma^{-1}$. The density increases with $\Gamma^{-1}$ for both small and large values of $\Gamma^{-1}$, as expected. However, there is a clear intermediate range in which the density decreases with $\Gamma^{-1}$, with a minimum of

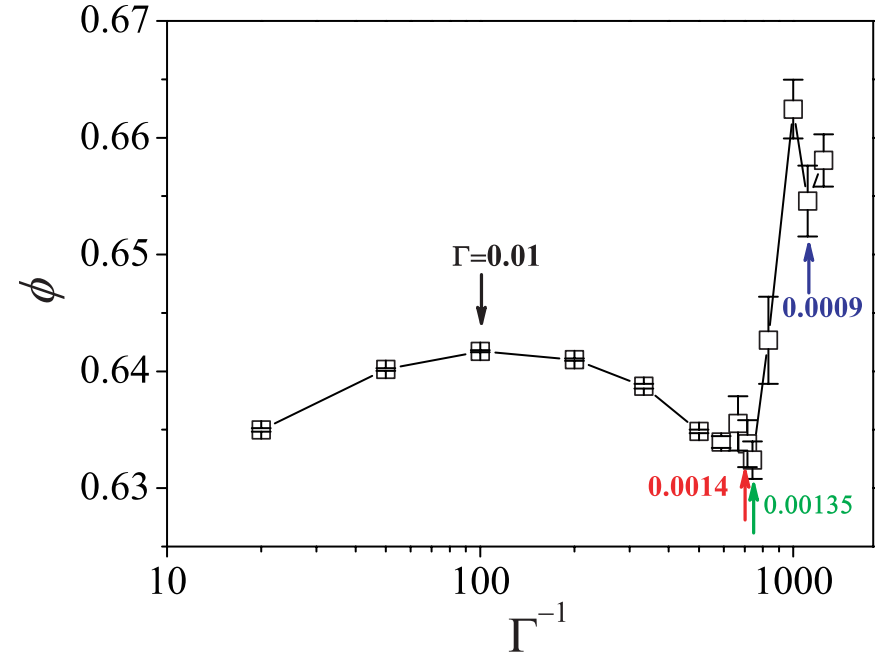

Fig. 1: (Color online) The terminal volume fraction of the structurally arrested state as a function of inverse of compression rate $\Gamma^{-1}$. All the structures obtained here are stable with no appreciable relaxation when left to equilibrate for prolonged period of time. The symbols represent the averages of 28 parallel samples formed by different initial conditions at each compression rate.

$\phi \approx 0.63$, occurring at $\Gamma \approx 0.00135$. We term states near this minimum the lower density arrested (LDA) states.

Analysis using the globally defined bond-orientation and translation order parameters shows increased order for the structures obtained with decreasing compression rate consistent with results from earlier work $[4,5]$. To characterize structural heterogeneity, we examine several locally defined metrics. We first consider the local bondorientation order parameter $Q_{6, j}^{L}[9,10]$ defined as

$$
Q_{6, j}^{L}=\left(\frac{4 \pi}{13} \sum_{m=-6}^{6}\left|\frac{1}{N_{b}^{j}} \sum_{\text {bond=1 }}^{N_{b}^{j}} Y_{6 m}\right|^{2}\right)^{1 / 2},
$$

where $Y_{l m}$ is the spherical harmonics, with $l=6$ in this calculation, and $N_{j}^{b}$ is the number of bonds for the $j$-th sphere, which is obtained by counting all the spheres lying within the minimum after the first peak in the radial distribution function. The average of $Q_{6}^{L}$ shows a behavior similar to the globally defined bond-orientation order parameter, and increases monotonically with decreasing compression rate. The more revealing result, however, is the distribution of $Q_{6}^{L}$. For common crystal structures such as the face-centered cubic (FCC), the hexagonal close-packed (HCP), and the body-centered cubic (BCC) phases, the distribution is sharply peaked with a peak position determined by the crystal lattice and a narrow spread due to thermal fluctuation. For structurally disordered states, the distribution is considerably wider. In fig. 2, we show the distribution of $Q_{6}^{L}$ for structures obtained at four different compression rates (corresponding to $\Gamma=0.01,0.0014,0.00135$, and 0.0009 ). 


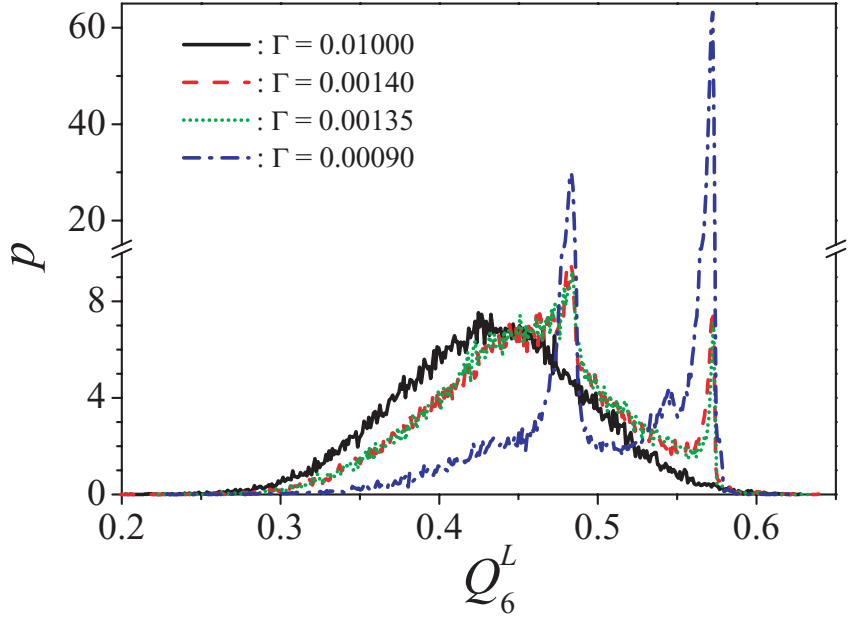

Fig. 2: (Color online) The distribution of the local bondorientation order parameter $Q_{6}^{L}$ in structurally arrested states generated by the following compression rates: (top to bottom) $\Gamma=0.01,0.0014,0.00135$ and 0.0009 . The distribution is obtained using all 28 parallel samples, each with 2048 particles.

The distribution for $\Gamma=0.01$ clearly corresponds to a glass-like structure. For the two LDA states obtained at intermediate compression rate $(\Gamma=0.0014$ and 0.00135$)$, there is more order as reflected by a shift of the mean to the right and a narrowing of the distribution. In addition, two peaks appear at $Q_{6}^{L} \approx 0.48$ and 0.57 , which reflects the presence of the HCP and FCC structures, respectively. These two peaks dominate the distribution at the smallest compression rate $\Gamma=0.0009$, with the FCC peak overtaking the HCP peak as the main peak; however, there is still a significant amount of background in the distribution due to noncrystalline structures. These results reflect the structural heterogeneity in the arrested states, the nature and degree of which are controlled by the compression rate. In particular, the LDA states contain amorphous regions as well as a mixture of HCP and FCC structures, which has been called the random hexagonally close-packed (RHCP) structure. Because of the small free energy difference between FCC and HCP [11-13], hard-sphere crystals usually contain both structures [14]. RHCP as an important metastable structure has also been reported in experiments [15] and computer simulations [16] in the nucleation in hard-sphere colloids. In our work these crystal domains form since the compression rates are such that the system has time to nucleate crystallites before reaching a glassy, structurally arrested state, but does not have time to allow the crystallites to grow because of continued compression.

Another signature of the structural heterogeneity comes from analyzing the distribution for the root-meansquare fluctuation $\left\langle(\Delta r)^{2}\right\rangle^{1 / 2}$ in the particle positions, which reflects the local mobility or free volume; in the supplementary material (see Suppl-material.pdf) for this letter, we show that this distribution can be used to

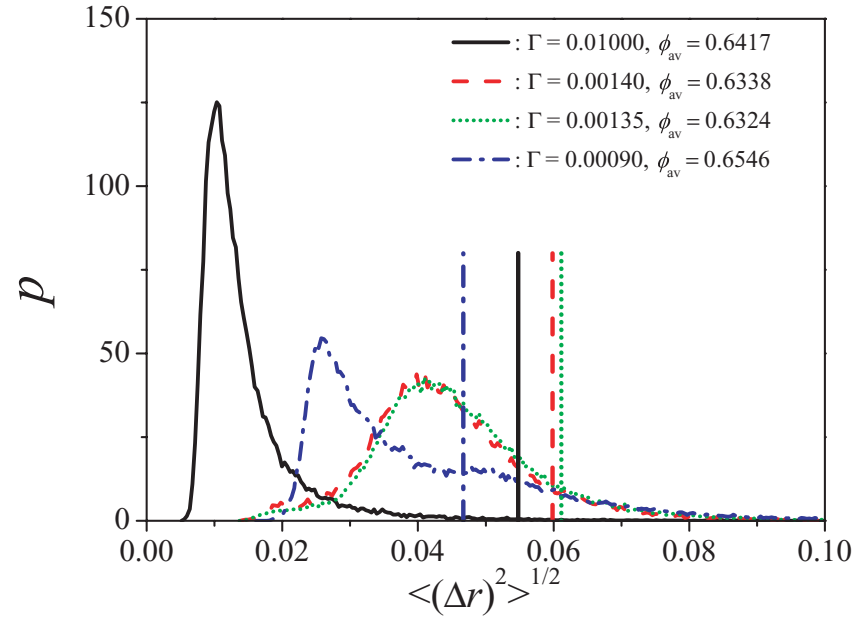

Fig. 3: (Color online) The distribution of the root-mean-square fluctuation $\left\langle(\Delta r)^{2}\right\rangle^{1 / 2}$ of the particle positions (scaled by the sphere diameter) at the same four compression rates as in fig. 2. The distribution is obtained using all 28 parallel samples, each with 2048 particles. For comparison, the corresponding value for the FCC crystal at the same density is indicated by the vertical bar of the same color.

estimate the free energy of the structurally arrested states $^{1}$. If all particles were in the same environments (as in perfect crystals), we would expect a single value of $\left\langle(\Delta r)^{2}\right\rangle^{1 / 2}$ for all the particles. In fig. 3 we show the distribution of $\left\langle(\Delta r)^{2}\right\rangle^{1 / 2}$ for the four structures obtained using the four different compression rate as in fig. 2. For comparison, the values for the FCC phase at the same corresponding densities are also included. For all cases there is considerable spread in the value of $\left\langle(\Delta r)^{2}\right\rangle^{1 / 2}$, and the average is less than the value for the FCC phase at the same density. While $\left\langle(\Delta r)^{2}\right\rangle^{1 / 2}$ for the perfect FCC crystal is clearly a monotonically decreasing function of the density, the average or peak position of the distribution for the structurally arrested states does not follow a simple correlation with the density. The most disordered state corresponding to the highest compression rate $\Gamma=0.01$ has the peak at the smallest $\left\langle(\Delta r)^{2}\right\rangle^{1 / 2}$, reflecting the more restricted local motion in the glassy state. On the other hand, the average of the distribution is not simply correlated with the overall degree of order either, since the most ordered state corresponding to the lowest compression rate $\Gamma=0.0009$ has its average below that of the two LDA states. This distribution thus provides a simple independent measure of the structural heterogeneity.

To obtain direct visual information on the crystalline regions, we use an algorithm developed by ten Wolde and Frenkel [17] for identifying particles belonging to a crystalline domain. The scalar product of local bondorder parameter defines a measure of the coherence of orientation for different particles. If a particle has more

\footnotetext{
${ }^{1}$ See online supplemental material for estimating the free energy of the jammed structures using the distribution of the root-meansquare fluctuation of the particle positions.
} 
a

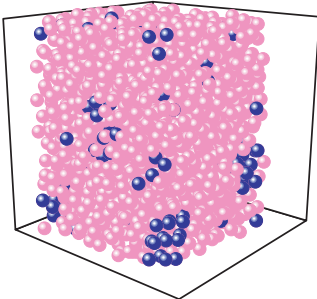

$\mathrm{C}$

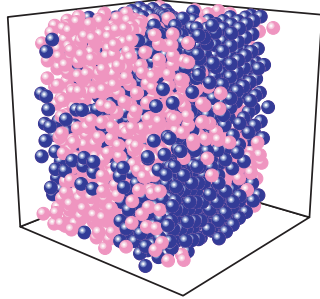

b

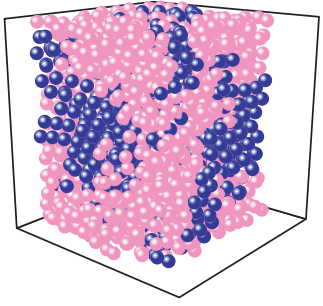

d

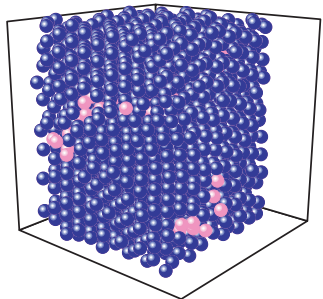

Fig. 4: (Color online) Snapshots of structurally arrested states obtained at (a) $\Gamma=0.01$, (b) 0.0014 , (c) 0.00135 , and (d) 0.0009. The dark (blue) spheres represent crystal-like particles and light (pink) spheres represent particles in amorphous regions.

than seven coherent neighbors, this particle will be defined as crystal-like. Two crystal-like spheres are considered to belong to the same crystal cluster if their distance is less than 1.25 sphere diameter. In the supplementary material, we provide a figure showing the number of crystal-like spheres and the number of clusters, the former increasing and the latter decreasing, as compression rate decreases ${ }^{2}$. The glassy states obtained using the highest compression rate contain more than 20 clusters and those clusters are small. The more crystalline states corresponding to the lowest compression rate consist of a single large cluster which contains the majority of spheres in the system. The LDA states contain several clusters, and the majority of the crystal-like spheres seem to belong to the largest cluster. In fact, the onset of the LDA states appears to coincide with the dominance by the largest cluster. Because our cluster criterion gives no information about the compactness of the cluster, the cluster size data does not distinguish between a large compact crystalline domain and several smaller connected locally ordered regions. However, visual images of the color-coded snapshots of the structure show that the large clusters in the LDA states (fig. 4b and c) consist of several small crystal clusters connected with each other that form a crystal "network". Therefore, the crystal clusters in these LDA states possess considerable amount of interfaces with the amorphous part of the structure. The packing of the spheres in these interfacial regions is even less efficient than that in the truly glassy state because of the additional frustration due to mismatch in the crystal orientation and shape irregularity. When the number of these highly frustrated particles is comparable to the number of spheres in the crystalline regions, the resulting structure can

\footnotetext{
${ }^{2}$ See online supplemental material for an analysis of crystal-like spheres and the number of crystal-like clusters.
}

have a lower density than that of the glassy states. Our results clearly demonstrate that the arrested structure for hard-spheres is inherently heterogeneous, consisting of amorphous and crystal-like domains. These crystal-like domains form probably because of fast nucleation kinetics. However, their growth becomes arrested as a result of continued compression. The higher the compression rate, the earlier their growth becomes arrested, resulting in many small crystal-like clusters interspersed in a largely amorphous structure at the higher compression rates. A reduced compression rate allows more time for the nuclei to grow further before becoming arrested. The evolution of the final arrested structure as a function of the inverse compression rate is therefore that of many small clusters increasing in size, touching each other to form a percolating network of polycrystalline domains, and eventually filling up the entire space. The increased order, as measured by the average bond-orientation and translation order shown in earlier work $[4,5]$ and in our present work, is in large part due to the increased fraction of the crystal-like domains, as opposed to increased order for each particle. The LDA states obtain near the onset of the percolation by the crystal clusters, where a large fraction of particles resides in the interfacial region, and the interlocking of the crystal clusters gives rise to very low packing efficiency for these particles.

In addition to the structural heterogeneity in arrested hard spheres, our work shows that structural arrest can involve multiple length scales: at the fastest compression rate arrest occurs on the particle length scale, while intermediate compression rate can lead to arrest by domains. Indeed, this two-scale picture offers a simple explanation of the trend in fig. 1. Because arrest occurs on the particle scale for fast compression, decreasing the compression rates leads to better local order and hence more efficient packing. This trend continues until a significant number of crystal clusters can nucleate at some lower compression rate. Decreased compression rate leads to larger clusters, but at the small cluster sizes, the growth of interfacial regions dominates over the growth in the volume of the clusters, and the low packing efficiency of these interfacial particles results in overall reduced packing density. The minimum density states correspond to the largest number of such interfacial particles, which as discussed above most likely occurs as the crystal-like clusters interlock to form a percolation network. Beyond this point, further decrease in the compression rate leads to larger cluster sizes and hence smaller number of interfacial particles, resulting in an increase in density accompanied by increased degree of order. Our work further suggests that there is no clear distinction between a glassy state and a polycrystalline state - both are part of a continuous spectrum that can be controlled by the compression rate. Since the compression rate for the athermal hard-sphere system can be related to the isobaric cooling rate [18] in liquid with attractive interactions, our findings and conclusions are likely to hold for other systems, such as Lennard-Jones particles. 
This work is supported by the National Natural Science Foundation of China (20574070, 20734003, 20620120105) Programs and the Fund for Creative Research Groups (50621302). ZYS also acknowledges the financial support of JLSTP (20070113).

\section{REFERENCES}

[1] Debenedetti P. G. and Stillinger F. H., Nature, 410 (2001) 259.

[2] Palberg T., J. Phys.: Condens. Matter, 11 (1999) R323.

[3] Liu A. J. and Nagel S. R., Nature, 396 (1998) 21.

[4] Torquato S., Truskett T. M. and Debenedetti P. G., Phys. Rev. Lett., 84 (2000) 2064.

[5] Truskett T. M., Torquato S. and Debenedetti P. G., Phys. Rev. E, 62 (2000) 993.

6] Lubachevsky B. D. and Stillinger F. H., J. Stat. Phys., 60 (1990) 561.
[7] Torquato S. and Stillinger F. H., J. Phys. Chem. B, 105 (2001) 11845.

[8] Donev A., Torquato S., Stillinger F. H. and Connelly R., J. Comput. Phys., 197 (2004) 139.

[9] Steinhardt P. J., Nelson D. R. and Ronchetti M., Phys. Rev. B, 28 (1983) 784.

[10] Kansal A. R., Truskett T. M. and Torquato S., J. Chem. Phys., 113 (2000) 4844.

[11] Woodcock L. V., Nature, 385 (1997) 141.

[12] Bolhuis P. G., Fenkel D., Mau S. C. and Huse D. A., Nature, 388 (1997) 235.

[13] Bruce A. D., Wilding N. B. and Ackland G. J., Phys. Rev. Lett., 79 (1997) 3002.

[14] Zhu J., Li M., Rogers R., Meyer W., Ottewill R. H., StS-73 Space Shuttle Crew, Russel W. B. and Chaikin P. M., Nature, 387 (1997) 883.

[15] Gasser U., Weeks E. R., Schofield A., Pusey P. N. and Weltz D. A., Science, 292 (2001) 258.

[16] Auer S. and Frenkel D., Nature, 409 (2001) 1020.

[17] ten Wolde P. R., Ruiz-Montero M. J. and Frenkel D., J. Chem. Phys., 104 (1996) 9932.

[18] Speedy R. J., Mol. Phys., 83 (1994) 591. 